\title{
Stereopsis and aniseikonia in uniocular aphakia
}

\author{
V. N. HIGHMAN \\ From Charing Cross Hospital, London W6 8RF
}

SUMMARY Uniocular aphakia in the adult when corrected with a contact lens is in most patients no obstacle to binocular function. The long-term result is a balance between binocular function obtained and the comfort of the contact lens, and in this respect the advent of the soft lens, and especially the continuous-wear lens, appears to be most promising.

Over the past five years 46 patients attending Charing Cross Hospital with uniocular cataracts have been treated surgically and the vision has been corrected in the aphakic eye with a contact lens. The clinical impression gained was that the results were good, and all those patients that could be contacted were re-examined.

The aniseikonia in uniocular aphakia, when corrected with spectacles, is of such a magnitude that binocular function is severely interfered with. Various attempts have been made to reduce the aniseikonia. Thus Eggers (1937) showed that it was possible to reduce aniseikonia by inducing spectacle magnification in the phakic eye and reducing it in the aphakic eye by suitably varying the back vertex distance of the correcting spectacle lens. When substituting a contact lens for a spectacle lens the reduction in the spectacle magnification reduces the image size (Treissman and Plaice, 1946), as the nodal point of the system, contact lens and eye, moves back. Ogle et al. (1958) showed that the residual aniseikonia does not appear to be an obstacle to fusion. Although aniseikonia may vary from 4 to $10 \%$, central or peripheral fusion can still be present, and the ability to fuse images when there is considerable difference in size may depend on the width of Pannum's areas (Burian, 1962). An alternative to the contact lens is the intraocular lens implant, which would be expected to reduce the aniseikonia even more than a contact lens, but even so it has been found that the aniseikonia may vary from $4 \%$ smaller to $6 \%$ larger in the aphakic eye and that the ability to fuse may also be variable, indicating that these appliances have little advantage over a contact lens (Troutman, 1962a, 1962b, 1963), although this point of view is contested by Choyce

Address for reprints: Mr V. N. Highman, Stoke Mandeville Hospital Aylesbury, Bucks.
(1964). The results of treating children with uniocular aphakia with contact lenses is disappointing (Daniel, 1974; Binkhorst and Gobin, 1967), as too many children respond with suppression and squint. The absence of accommodation in the aphakic eye may play a part as suppression was more common in the younger rather than in the older children (Daniel, 1974).

\section{Method}

Patients were refracted with the contact lens in situ so that any residual astigmatism associated with the contact lens could be corrected and the best possible correction given to the other eye. All tests were then carried out with a trial frame containing the appropriate correcting lenses. Aniseikonia was determined by means of Ruben's aniseikonic plates and slides and the results were expressed as a percentage. Stereopsis was then measured with the Titmus Stereo-circles Chart, lenses were added to the trial frame to correct for the reading distance when required, and special care was taken to ensure that true stereopsis was being experienced rather than just blurring of the circles, which may occur at the $400^{\prime \prime}$ and $800^{\prime \prime}$ separations. The results were expressed as log stereopsis.

A group of 30 patients with different degrees of myopia but no other abnormality, all of whom had stereopsis, were used as a control group to determine if there was any relationship between stereopsis and aniseikonia. Aniseikonia was induced by correcting the less myopic eye with a soft contact lens (Sauflon 70) and the more myopic eye with the appropriate correcting lens in a trial frame. Any residual astigmatism associated with the contact lens was also corrected with the appropriate cylindrical lens in the trial frame. By suitable selection of myopes of varying severity a wide range of aniseikonic measure- 
ments were obtained, as well as the stereopis for each respective case. All subjects had a visual acuity of $6 / 9$ or better in each eye when corrected.

A third group of unselected patients attending for routine refraction were examined to determine whether stereopsis changed with age. There were two groups-30 patients aged 20 to 30 years and 30 patients aged 60 to 70 , all with a corrected visual acuity of $6 / 9$ or better, in each eye.

\section{Results}

An analysis of the contact lenses used during the period under investigation showed: Hard lenses 2; soft lenses 7 ; continuous wear 18 ; total 27 . All of the 27 patients investigated had worn their lenses for at least six months, and no hard lenses were fitted after 1972. The continuous-wear lenses were all fitted in the last 18 months of the period. In those patients wearing the continuous-wear soft lenses no significant change was noted in aniseikonia or stereopsis over a three-month period. In all the patients a visual acuity of $6 / 9$ in each eye was obtained.

In the myopic group of subjects a significant correlation was found between per cent aniseikonia and $\log$ stereopsis (Fig. 1). The regression line for $\log$ stereopsis on per cent aniseikonia, by the theorem of least squares, is drawn as a solid line; on either side of the regression are the $95 \%$ confidence limits in dotted lines, the inner lines for the regression line and the outer for the individual points. Aniseikonia greater than $19 \%$ was not obtained.

It was possible to measure aniseikonia in all 27 uniocular aphakics, but in two there was no demonstrable stereopsis on the Titmus Chart, circles or fly. The aniseikonia in these two patients was 25 and $29 \%$ respectively. In all the other patients the aniseikonia was below $20 \%$.

No significant difference was found between the mean stereopsis for the two age groups, 20 to 30 years and 60 to 70 years.

The continuous-wear lenses were made of Sauflon 85 (poly (vinyl pyrrolidone $\times$ methyl methacrylate) $85 \%$ ); of the daily wear lenses five patients had hydron lenses (polyhydroxyethyl methacrylate), and in two patients Sauflon 70 (poly (vinyl pyrrolidone $x$ methyl methacrylate) $70 \%$ ).

\section{Discussion}

In the myopic group of subjects on the assumption that per cent aniseikonia is the independent value and $\log$ stereopsis the dependent it can be seen from Fig. 1 that an empirical relationship does exist

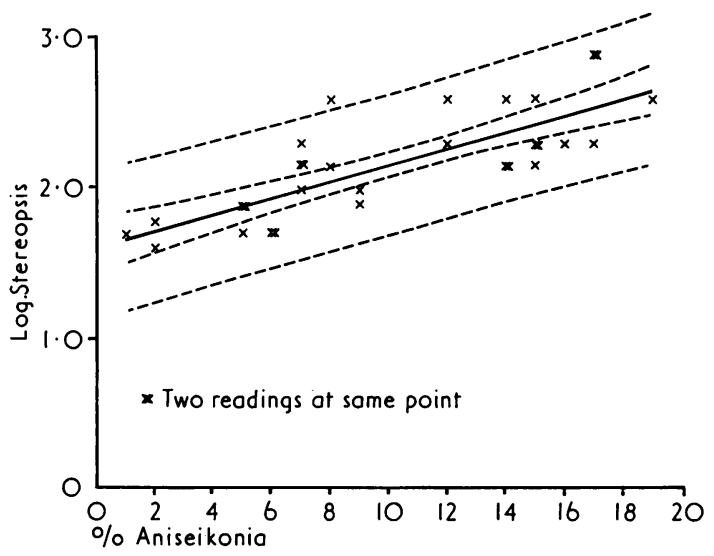

Fig. 1 Log stereopsis plotted against induced per cent aniseikonia for the control of myopic group of subjects. The straight-line relationship is shown as a solid line, and the $95 \%$ confidence limits are shown as dotted lines on either side of the regression line. The outer dotted lines are the $95 \%$ confidence limits for the individual points

between the two, the correlation coefficient being $0 \cdot 78$. The results obtained were used to determine whether the uniocular aphakic group of patients compared in any way. It was initially hoped that a relationship between per cent aniseikonia and log stereopsis would be found in the uniocular group, but this was not the case, possibly because the aniseikonic measurements were bunched together. When the results from the uniocular aphakics are statistically tested against the myopic group it may be concluded that they come from the same statistical population (Fig. 2).

Stereopsis was present up to an aniseikonia of $19 \%$, but above this value central stereopsis probably breaks down, for in the two uniocular aphakic patients the aniseikonia was 25 and $29 \%$ respectively and no stereopsis was present on the Titmus Stereocircle test. This is to be expected, for when looking at the results obtained from the myopic control group (Fig. 1) when aniseikonia is approaching $20 \%$ at best only a $400^{\prime \prime}$ of an arc separation would be expected when testing on the Titmus Stereo-circle test. On fitting contact lenses diplopia was initially experienced in some of the uniocular aphakics but persisted only for some 24 hours. No improvement in aniseikonia or stereopsis was observed in any of the patients after wearing the lenses for three months.

One patient, a professional dancer with uniocular aphakia, was of particular interest. He had been unable to cope with his daily wear contact lens, for while dancing he experienced spacial disorientation 


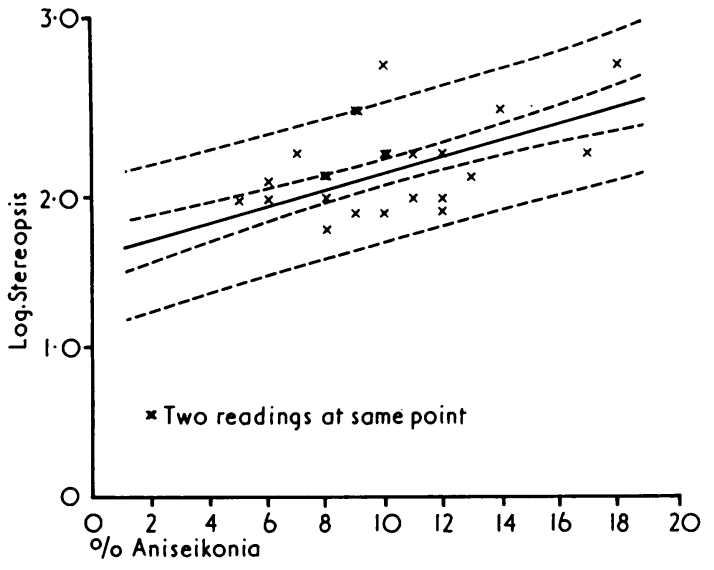

Fig. 2 Results obtained from the uniocular aphakic group superimposed upon the regression line and $95 \%$ confidence limits obtained from the control myopic group of subjects

owing to tilt of the horoptor. Between engagements advantage was taken to fit a continuous-wear contact lens, tilt of the horoptor was again initially noted, but within 24 hours he had adapted to his $14 \%$ aniseikonia, and spacial distortion was no longer experienced.

From Fig. 2 it may be seen that most of the results are bunched around an aniseikonia of $10 \%$, which gives a very useful degree of stereopsis. Even the two patients without central stereopsis had some degree of binocular function and benefited from the improved peripheral field on the aphakic side. Thus, provided stereopsis was present prior to development of the cataract the results indicate that useful stereopsis will be present in most patients when correcting with a contact lens. The time of fitting does not appear to be important, as patients were fitted from two months to three years after surgery, though it must be pointed out this group of patients consisted only of adults. The results confirm a previous report (Nolan et al., 1975) that it is worth considering a routine contact lens trial after cataract extraction and leaving the insertion of an intraocular lens implant as a secondary procedure.

Of interest is the trend towards soft contact lenses, as experience has shown that these are more comfortable and can be worn for longer periods than hard lenses. Even more important is the development of hydrophylic lenses with a manufacturer's claim of high oxygen permeability in order to permit continuous wear. With the evolution of more comfortable lenses it has now become apparent that a balance exists between the visual benefit obtained with a contact lens and the bother of handling, wearing, and caring for a lens. This is most clearly demonstrated when using continuous-wear lenses, as patient handling is eliminated and comfort is at an optimum. Further, as most soft lenses are a semi-scleral fit, stability is greater than with a small hard corneal lens, so reducing the prismatic effect that is commonly encountered. It would appear to be the rule that patients become readily accustomed to quite high degrees of aniseikonia, given a troublefree contact lens.

Today there should be little hesitation in removing a uniocular cataract in the adult. It is suggested that after surgery there should be a contact lens trial, and the author's preference is to fit a soft rather than a hard contact lens provided the aniseikonia is below $20 \%$. The type of lens chosen will depend on the patient's ability to handle a daily wear lens. In the elderly, or when the aniseikonia is greater than $14 \%$, a continuous-wear lens is indicated as first choice. When the aniseikonia is greater than $20 \%$ a continuous-wear lens may be tried, but a patient in this category is probably a candidate for an intraocular lens implant provided binocular function is present. Continuous-wear lenses are not without complications (Highman, 1975). Thus out of 53 aphakic lenses thus fitted there has been corneal vascularisation ( 1 case), infection ( 1 eye), microcystic oedema ( 1 eye), and an 'acute red eye reaction' ( 5 cases). In spite of these complications none of the patients have been permanently stopped wearing their lenses. In children there would seem to be a case for overcorrecting by +3 dioptres and giving spectacles for distance, because of the absence of accommodation.

I should like to thank Mr Arnott and Mr Higgitt for their help in allowing me to examine their patients for the above investigation. I should also like to thank the Governors of Charing Cross Hospital for their financial help and the Medical Illustration Department for the art work.

\section{References}

Binkhorst, C. D., and Gobin, M. H. (1967). Pseudophakia after lens injury in children. Ophthalmologica, 154, 81-87.

Burian, H. M. (1962). Optics: Fusion in unilateral aphakia. Transaction of the American Academy of Ophthalmology and Otolaryngology, 66, 285-9.

Choyce, P. (1964). Intraocular Lenses and Implants. London, H. K. Lewis.

Daniel, R. (1974). An evaluation of contact lenses in unilateral post-traumatic aphakic children. Contact Lens, 4, 19-24.

Eggers, H. (1937). Effect of position of correcting lens on size of retinal image. Archives of Ophthalmology, 17, 328339.

Highman, V. (In press). Correction of aphakia with contact lenses. Proceedings of the Symposium on Bio-engineering 
in Ophthalmology. Haifa, Israel, 1975. Documenta Ophthalmologica.

Nolan, J., Hawkswell, A., and Becket, S. (1975). Fusion in aphakia. Proceedings of the Third International Orthoptic Congress. Boston, Mass., USA.

Ogle, K. N., Burrian, H. M., and Bannon, R. E. (1958). On the correction of unilateral aphakia with contact lenses. A.M.A. Archives of Ophthalmology, 59, 639-652.

Ruben, M. (1962). Unilateral aphakia. British Orthoptic Journal, 19, 39-61.
Treissman, H., and Plaice, E. A. (1946). The Principles of the Contact Lens. London, Kimpton.

Troutman, R. C. (1962a). Artiphakia and aniseikonia. Transactions of the American Ophthalmological Society. 60, 590-658.

Troutman, R. C. (1962b). Correction of unilateral aphakia. The use of intraocular lens implants. Archives of Ophthalmology, 68, 861-9.

Troutman, R. C. (1963). Artiphakia and Aniseikonia. American Journal of Ophthalmology, 56, 602-639. 